This document is the accepted manuscript version of the following article:

Pan, D., Hufenus, R., Qin, Z., Chen, L., \& Gooneie, A. (2019). Tailored gradient morphologies and anisotropic surface patterns in polymer blends. Macromolecular Materials and Engineering, 304(3), 1800601 (12 pp.).

https://doi .org/10.1002/mame. 201800601

\title{
Tailored Gradient Morphologies and Anisotropic Surface Patterns in Polymer Blends
}

\author{
Dan Pan ${ }^{\mathrm{a}, \mathrm{b}}$, Rudolf Hufenus ${ }^{\mathrm{a}}$, Zongyi Qin ${ }^{\mathrm{b}}$, Long Chen ${ }^{\mathrm{a}, \mathrm{b} *}$, Ali Gooneie ${ }^{\mathrm{a} *}$ \\ ${ }^{a}$ Laboratory of Advanced Fibers, Empa, Swiss Federal Laboratories for Materials Science \\ and Technology, Lerchenfeldstrasse 5, CH-9014 St. Gallen, Switzerland \\ ${ }^{\mathrm{b}}$ State Key Laboratory for Modification of Chemical Fibers and Polymer Materials, \\ College of Materials Science and Engineering, Donghua University, Shanghai 201620, \\ P.R.China
}

*Corresponding Authors: (A.G.) ali.gooneie@empa.ch; (L.C.) happyjack@ dhu.edu.cn

\begin{abstract}
Gradient morphologies and surface patterns can provide a template for smart materials with tailored local properties. Fabricating such structures in a one-step meltextrusion process would be most beneficial as they usually require several manipulation steps. In this study, we develop gradient morphologies by melt-spinning matrix-dispersed polypropylene/polystyrene blends as a template system. We achieve dynamic control of their formation which further leads to anisotropic surface patterns. Cross and longitudinal sections, and the surface of extruded fibers, are evaluated by scanning electron microscopy. Distinct microstructure gradients regarding size and shape of the dispersed phase are formed in the bulk. After dissolving the dispersed phase, ordered (sub)micron channels appear on the surface, which are further refined by hot-drawing. The resulting textures form anisotropic grooves along the fiber axis at the surface, as verified by atomic force microscopy. Capillary theory is applied to elucidate the formation mechanisms of gradient microstructures in
\end{abstract}


polymer blends during processing. Our investigations show that all these features can be tailor-made by using the extrusion die as a design tool. By transferring the knowledge to suitable polymer blends and processes, the designed gradient and surface structures can find applications, for instance, in materials with adaptive mechanical or interfacial properties.

Keywords: gradient morphology; polymer blends; surface pattern; melt processing.

\section{Graphical Abstract}

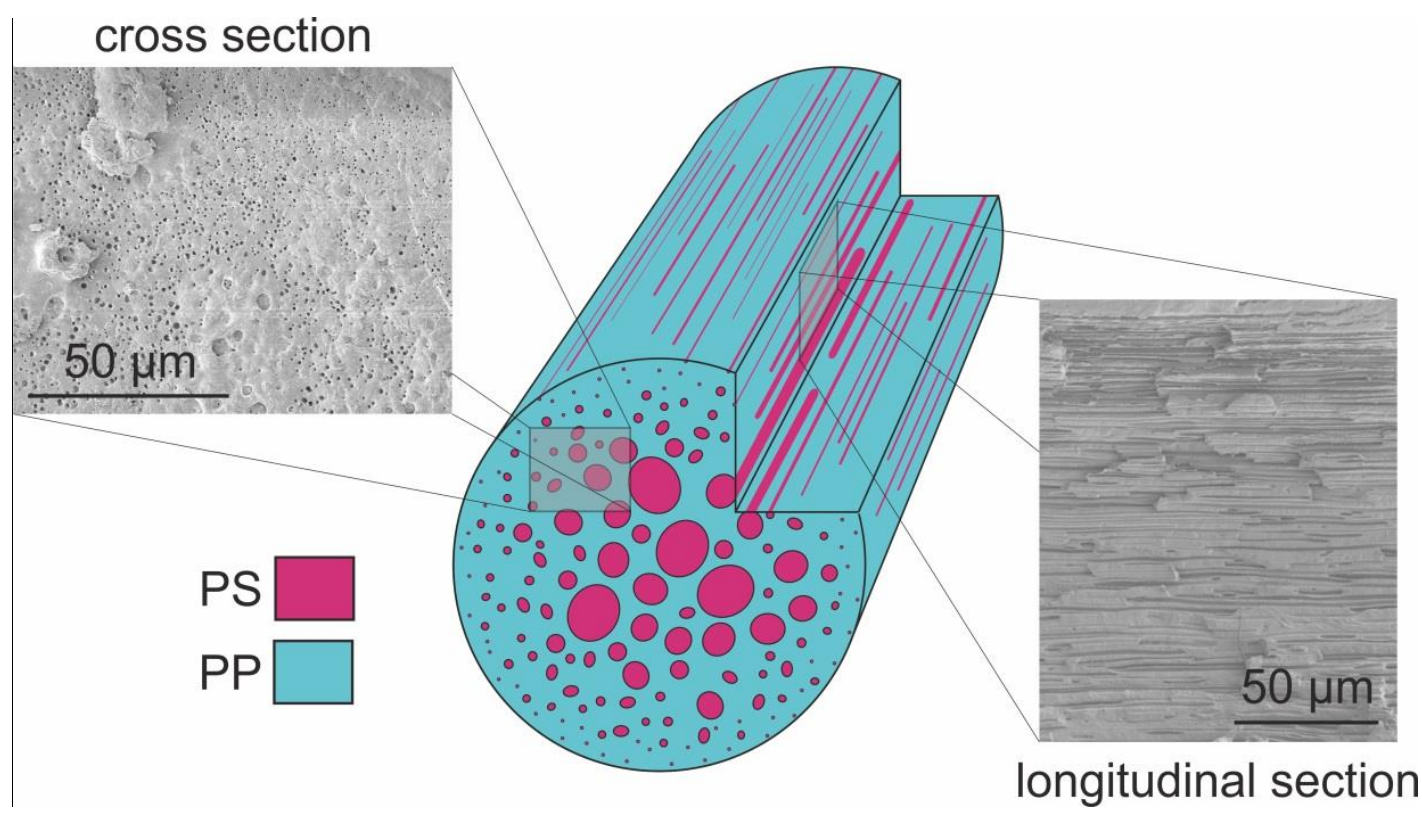




\section{Introduction}

Gradient polymer blends are truly attractive materials, since their particular morphology allows to develop desired properties in certain regions of the material. ${ }^{[1-3]}$ Such gradient structures have found elaborate applications, for instance in graded index optical fibers, where the refractive index of a polymer is controlled by the diffusion of immiscible monomer species. ${ }^{[4-6]}$ The impact of a gradient morphology is not necessarily limited to the bulk, but can also influence the surface properties. For instance, the surface tension of polypropylene (PP) fibers can be modified with a gradient morphology if the polystyrene (PS) dispersed phase is locally stretched at the surface of PP/PS blends. This type of gradient structures has in particular found applications in the coloring of PP fibers and textiles. ${ }^{[7]}$ Gradient structures are also beneficial to fulfill requirements for biological and anatomical applications, owing to their different features embodied in different layers. For instance, functional gradient tissue engineering scaffolds have been utilized to facilitate the regeneration of failing human tissues and organs. ${ }^{[8,9]}$ Despite these alluring features, the full potential of gradient morphologies is yet to be exploited by a dynamic control of the dispersed phase transformations during material formation and processing.

Gradient morphologies have been investigated in previous studies with the goal to design smart materials. The current approach to design a gradient morphology is mainly focused on post-processing by thermal annealing ${ }^{[2,10-12]}$ or in electric fields. ${ }^{[13]}$ Microphase separation in solvent-cast films is also reported to produce a gradient structure (of compositions) in polymer blends. ${ }^{[14,15]}$ However, a more desirable approach would be to dynamically achieve such gradients during fabrication, by controlling the process parameters. Some efforts have 
been made towards in-situ preparation of gradient polymer blends by controlling composition, viscosity ratio, and stress field during processing. ${ }^{[7,16]}$ Nevertheless, a comprehensive design strategy for such materials is still far from realization. The elaborate use of micro- and nanoparticles to influence the morphology of polymer blends has also been investigated. ${ }^{[16-19]}$ It is widely accepted that the morphology of polymer blends is mainly governed by the deformation, migration, breakup and coalescence of the dispersed phase domains. ${ }^{[16,20-28]}$ These phenomena, in turn, are controlled by the processing conditions, and are well addressed in numerous rheological studies. Among all approaches to investigate polymer blends, the processing of polymeric fibers is particularly attractive, since it provides a dynamic and adjustable platform for both elongational and shear flows. ${ }^{[29]}$ So far, most of the research in this area is directed towards the understanding of elongational forces, and their role in the development of fibril and gradient patterns at the micro- or nanoscale. ${ }^{[7,30-}$ ${ }^{33]}$ In this study, we discuss evidence showing the additional role of shear forces on the generated gradient structures, in the presence of controlled elongational forces.

An important difference of dynamic formation of gradient microstructures in a capillary die (during processing), and the conventional thermal annealing method (post-processing) is as follows: In the former, the gradient size of the dispersed phase can be decreasing from the bulk to the surface of the material (by design), whereas the reverse is true in the latter. ${ }^{[2,34]}$ It has further been shown that the size gradient depends on the interfacial tension of the blends. ${ }^{[35]}$ In a gradient morphology generated by annealing, the spatial temperature gradient has a significant effect on the droplet shape. ${ }^{[36]}$ Similarly, we will show here that the morphology development driven by velocity gradients in a capillary die allows controlling 
the shape of droplets, changing them from large spheres to elongated fibrils. A shear-induced gradient morphology can offer additional flexibility regarding surface modification of a blend. Here, multicomponent anisotropic surfaces are developed where the dispersed phase is present at the surface and oriented in flow direction. Towards the end of this paper, we show that such patterns are initiated merely by shear flows in a continuous process, but can be enhanced by elongational flows.

A more elaborate use of this gradient structure can be the engineering of sub-micrometer hierarchical textures and microchannels. In a gradient fiber, the thin near-surface fibrils of the second phase can be dissolved and washed off in a post-processing step. Therefore, one can achieve submicron (even nano-sized) patterns at the fiber surface by a proper design of shear/elongational flows. Recent studies on preform-to-fiber thermal drawing have introduced a discontinuous technique to produce such submicron patterned fibers. ${ }^{[37]}$ For instance, fibers with surface patterns have shown improved moisture management properties. ${ }^{[38]}$ More exclusively, such designed textures can find applications in thin-film photovoltaic cells, ${ }^{[39]}$ or act as a template support for directed growth of cells and for regeneration of nerves in the human body. ${ }^{[37,40-42]} \mathrm{A}$ continuous production of fine surface patterns has the potential to replace existing lithographic techniques, ${ }^{[43]}$ as well as dewetting approaches. ${ }^{[44,45]}$

In this paper, we attempt to provide a comprehensive picture of how shear flows lead to gradient morphologies in PP/PS blend fibers. Our investigation comprises a series of capillary dies with variant aspect ratios (ARs) in the presence of elongational forces controlled via take-up speeds. Bulk gradient morphologies and surface patterns are 
characterized in terms of size and shape of the dispersed PS domains. We provide evidence that an extrusion die can be used as a modulating design unit to continuously induce gradient morphologies within a polymer blend. Finally, we show that the incorporated processing protocols can lead to the formation of anisotropic patterns on the surface of samples.

\section{Experimental Details}

\subsection{Materials}

Polypropylene (PP, HSP165G) with melt flow index of $16.5 \mathrm{~g} / 10 \mathrm{~min}\left(230^{\circ} \mathrm{C} / 2.16 \mathrm{~kg}\right)$ was obtained from RESINEX Switzerland AG. Polystyrene (PS, STYRON 643) with melt flow index of $3.5 \mathrm{~g} / 10 \mathrm{~min}\left(200{ }^{\circ} \mathrm{C} / 5 \mathrm{~kg}\right)$ was obtained from Trinseo Europe $\mathrm{GmbH}$ (Switzerland).

\subsection{Rheological Measurements}

The rheological properties of the polymers were measured using a rotary rheometer (MCR 303, Anton Paar $\mathrm{GmbH}$ ) with a parallel plate fixture. The shear viscosity of each polymer was measured at $230{ }^{\circ} \mathrm{C}$. After loading the samples into the cell, temperature was maintained at $230{ }^{\circ} \mathrm{C}$ for $4 \mathrm{~min}$ to melt the polymers, and subsequently equilibrated for $30 \mathrm{~s}$ prior to the rheological measurements. Steady sweeps were performed with shear rates ranging from $10^{-}$

${ }^{2}$ to $10^{1} \mathrm{~s}^{-1}$. The strain amplitude sweeps were performed at an angular frequency of $10 \mathrm{rad} / \mathrm{s}$, followed by the frequency sweep tests. All rheological measurements were repeated three times to demonstrate reproducibility. 


\subsection{Sample preparation}

PP and PS granules were physically mixed in a mechanical mixer, and subsequently dried at $70{ }^{\circ} \mathrm{C}$ overnight under vacuum to minimize moisture content. The polymer mixture was melt-spun on a lab-scale fiber extrusion line with a single-screw extruder $(\mathrm{D}=19 \mathrm{~mm}$, $\mathrm{L} / \mathrm{D}=25$, Rheomex OS, Thermo Fisher Scientific, Germany) at a processing temperature of $220^{\circ} \mathrm{C}$. A static mixer was installed between extruder and melt pump (Haake OS, Thermo Fisher Scientific) to promote dispersion and distribution of PS. The as-spun monofilament was quenched in a water bath installed beneath the spinneret, with a distance between spinneret and water surface of $\mathrm{d}=5 \mathrm{~cm}$. The custom-made capillary dies all had a conical entrance zone with a cone angle of $2 \theta=26.5^{\circ}$ and a length of $\sim 30 \mathrm{~mm}$, as well as a diameter ( $\mathrm{D}_{\text {cap }}$ ) of $1.5 \mathrm{~mm}$. The morphology development of the polymer blend was studied by changing the length of the capillary die $\mathrm{L}_{\text {cap }}$ (hence changing $\mathrm{AR}=\mathrm{L}_{\mathrm{cap}} / \mathrm{D}_{\text {cap }}$ ) and the takeup speed $v$ of the spinning line. The investigated ARs were 2, 23, 37, and 57. The take-up speeds $\mathrm{v}$ were 10,20 , and $30 \mathrm{~m} / \mathrm{min}$. The schematic diagram of fiber melt extrusion is shown in Figure 1. In our process the die swell was negligible due to the dominance of take-up action in agreement with previous experiments and models. ${ }^{[46,47]}$ Table 1 summarizes the operating parameters of the different extrusion trials performed in the present study. Also, a complete list of all processing parameters is given in Table $\mathrm{S} 1$ of the Supporting Information. 


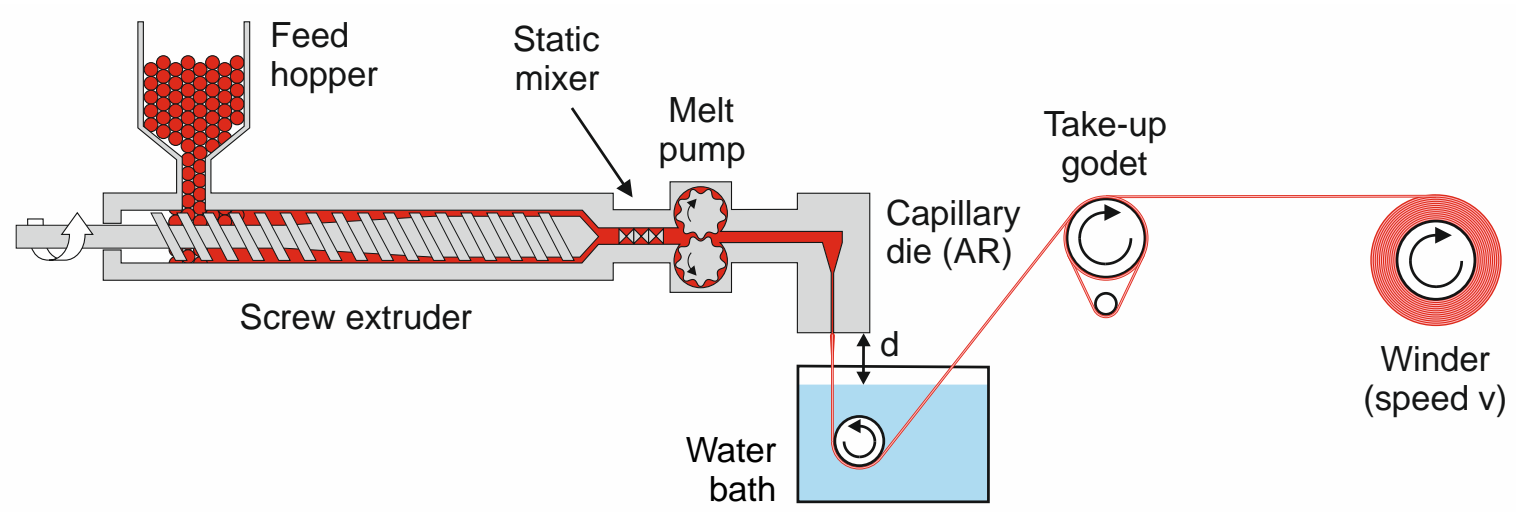

Figure 1. Schematic diagram of melt extrusion of PP/PS blend fibers.

Table 1. Processing conditions and residence times for polymer flows through extrusion line and capillary dies. $t_{1}$ is the residence time in the capillary, and $t_{2}$ is the residence time between spinneret and water bath.

\begin{tabular}{llllllll}
\hline $\begin{array}{l}\text { Capillary } \\
\text { AR }\end{array}$ & $\begin{array}{l}\text { Take up } \\
\text { speed } \\
{[\mathrm{m} / \mathrm{min}]}\end{array}$ & $\begin{array}{l}\text { Mass } \\
\text { flow rate } \\
{[\mathrm{g} / \mathrm{min}]}\end{array}$ & $\begin{array}{l}\text { Draw- } \\
\text { down } \\
\text { ratio } \\
\text { DDR }\end{array}$ & $\begin{array}{l}\text { Melt } \\
\text { temperatu } \\
\text { re in die } \\
{\left[{ }^{\circ} \mathrm{C}\right]}\end{array}$ & $\begin{array}{l}\text { Die } \\
\text { pressure } \\
{[\mathrm{bar}]}\end{array}$ & $\begin{array}{l}\text { Mean residence } \\
\text { times }\end{array}$ & \\
\hline \multirow{4}{*}{2} & 10 & 5.88 & 2.8 & 232 & 13 & 0.05 & 0.23 \\
& 20 & 5.88 & 5.6 & 232 & 13 & 0.05 & 0.13 \\
& 30 & 5.88 & 8.4 & 232 & 13 & 0.05 & 0.09 \\
& 10 & 5.55 & 3.0 & 229 & 41 & 0.67 & 0.23 \\
& 23 & 5.55 & 5.9 & 229 & 41 & 0.67 & 0.13 \\
& 20 & 5.55 & 8.9 & 229 & 41 & 0.67 & 0.09 \\
& 30 & 5.30 & 3.1 & 230 & 60 & 1.10 & 0.23 \\
& 10 & 5.30 & 6.2 & 230 & 60 & 1.10 & 0.13 \\
& 20 & 5.30 & 9.3 & 230 & 60 & 1.10 & 0.09 \\
& 30 & 5.20 & 3.2 & 239 & 86 & 1.70 & 0.23 \\
& 10 & 5.20 & 6.3 & 240 & 86 & 1.70 & 0.13 \\
& 20 & 5.20 & 9.5 & 239 & 86 & 1.70 & 0.09 \\
\hline
\end{tabular}

Hot drawing experiments were performed on a tensile testing machine (Z100, Zwick, Germany), equipped with an integrated temperature-controlled chamber. Fiber samples were maintained at $100{ }^{\circ} \mathrm{C}$ for approximately $3 \mathrm{~min}$ to reach temperature equilibrium. Subsequently, fibers were drawn at $100{ }^{\circ} \mathrm{C}$ with a crosshead speed of $500 \mathrm{~mm} / \mathrm{min}$ to an overall draw ratio of 5 (increase of grip distance from 5 to $25 \mathrm{~cm}$ ). 


\subsection{Morphology Characterization}

In order to study the morphology of blends, PP/PS blend fibers were carefully fixed in a holder with defined orientation, embedded in epoxy resin, and solidified at room temperature. The embedded samples were polished in a grinder polisher (EcoMet 250 Pro, Buehler, Illinois, USA) to obtain cross and longitudinal sections of fibers. To dissolve the dispersed PS phase at the surface, the polished samples were immersed in tetrahydrofuran (Analytical standard, Aldrich) under ultrasonic conditions for $10 \mathrm{~min}$, and then in ethanol under ultrasonic conditions for another $10 \mathrm{~min}$. This process was repeated three times at room temperature to wash away the dissolved part.

Scanning electron microscopy (SEM, S4800, Hitachi, Japan) and atomic force microscopy (AFM, FlexAFM, Nanosurf, Switzerland) were used to analyze bulk and surface morphologies of blend fibers. In case of atomic force microscopy (AFM), imaging was performed using the tapping mode with a probe of $2 \mathrm{~nm}$ nominal radius.

\subsection{Image Analysis}

Micrographs of the fiber sections were evaluated using image analysis functionalities in MATLAB ${ }^{[48]}$ First, SEM pictures were combined to reconstruct the entire diagonal of a fiber cross-section, as depicted in Figure 2. The image of such a fiber section was then divided into six partitions with equal width, in order to study the radial morphology difference. At least five different cross-sections per fiber sample were analyzed accordingly to ensure appropriate statistics. Typical morphology representations of fiber sections were processed by image analysis. Here, droplet sizes were enlarged 250 times and colored according to a 
defined scale bar. The droplets were plotted at their corresponding locations in the fiber section.

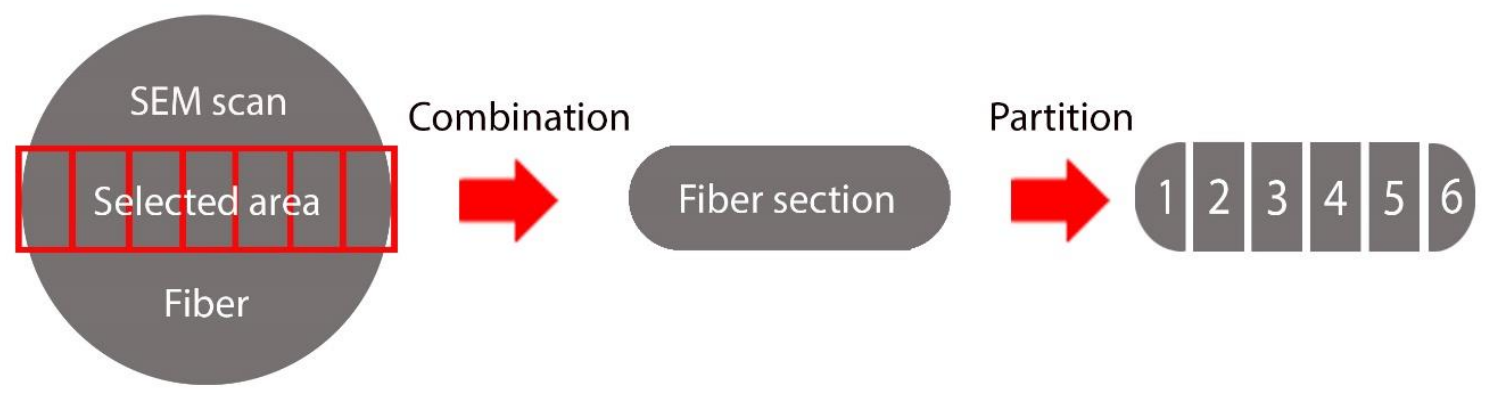

Figure 2. Combination and partitioning of micrographs of fiber cross-sections for analysis.

In image analyses, the droplet radius of the PS phase is defined as equivalent area radius:

$$
\mathrm{r}=\sqrt{\frac{\mathrm{A}}{\pi}}
$$

where $\mathrm{A}$ is the droplet area of the dispersed phase. The number probability $\mathrm{p}_{\mathrm{n}}$ of the dispersed phase is defined as the ratio between number of droplets in a defined radius range and the total number of droplets.

$$
\mathrm{p}_{\mathrm{n}}=\frac{\sum \mathrm{n}_{\mathrm{k}<i<j}}{\sum \mathrm{n}_{\mathrm{i}}}
$$

where $n_{i}$ is the number of dispersed phases with a radius of $r_{i}, n_{k<i<j}$ is the number of dispersed phases within the radius range of $r_{k}$ and $r_{j}$. The average radius of PS phases is defined as

$$
\overline{\mathrm{r}}=\frac{\sum \mathrm{n}_{\mathrm{i}} \mathrm{r}_{\mathrm{i}}}{\sum \mathrm{n}_{\mathrm{i}}}
$$

where $n_{i}$ is the number of dispersed phases with a radius of $r_{i}$. 


\section{Results and Discussions}

\subsection{Gradient Morphologies in PP/PS Blends}

The typical morphology of fiber cross-sections, resulting from melt extrusion trials with different capillary ARs and take-up speeds, is shown in Figure 3, where the black spots represent the dissolved PS domains. The corresponding analyzed morphology images of the fiber cross-sections are displayed in Figure 4. Small PS domains (shown in blue) are mostly concentrated close to the fiber surface. On the other hand, the relatively larger PS domains become more dominant towards the central axis of the fiber. Such a distribution creates a droplet size gradient in radial direction of the fiber in cross-sectional cuts. As indicated by Figure 4, the microstructure is influenced by the variations of capillary AR and take-up speed. Figure S1 of the Supporting Information shows the radial distribution of PS droplet radii in PP/PS blend fibers melt-spun using different capillary dies and taken up at different speeds. The results show, for all studied cases, that the average radii of the PS phase in the center region are larger than those in the surface region. As depicted in Figure S1, the average droplet radius decreases with increasing take-up speed and, to a lesser extent, with increasing capillary AR. As indicated by the results, an increase in elongational forces in terms of takeup speed slightly weakens the size gradient profile, i.e. uniforms the droplet radii along the radial direction. 


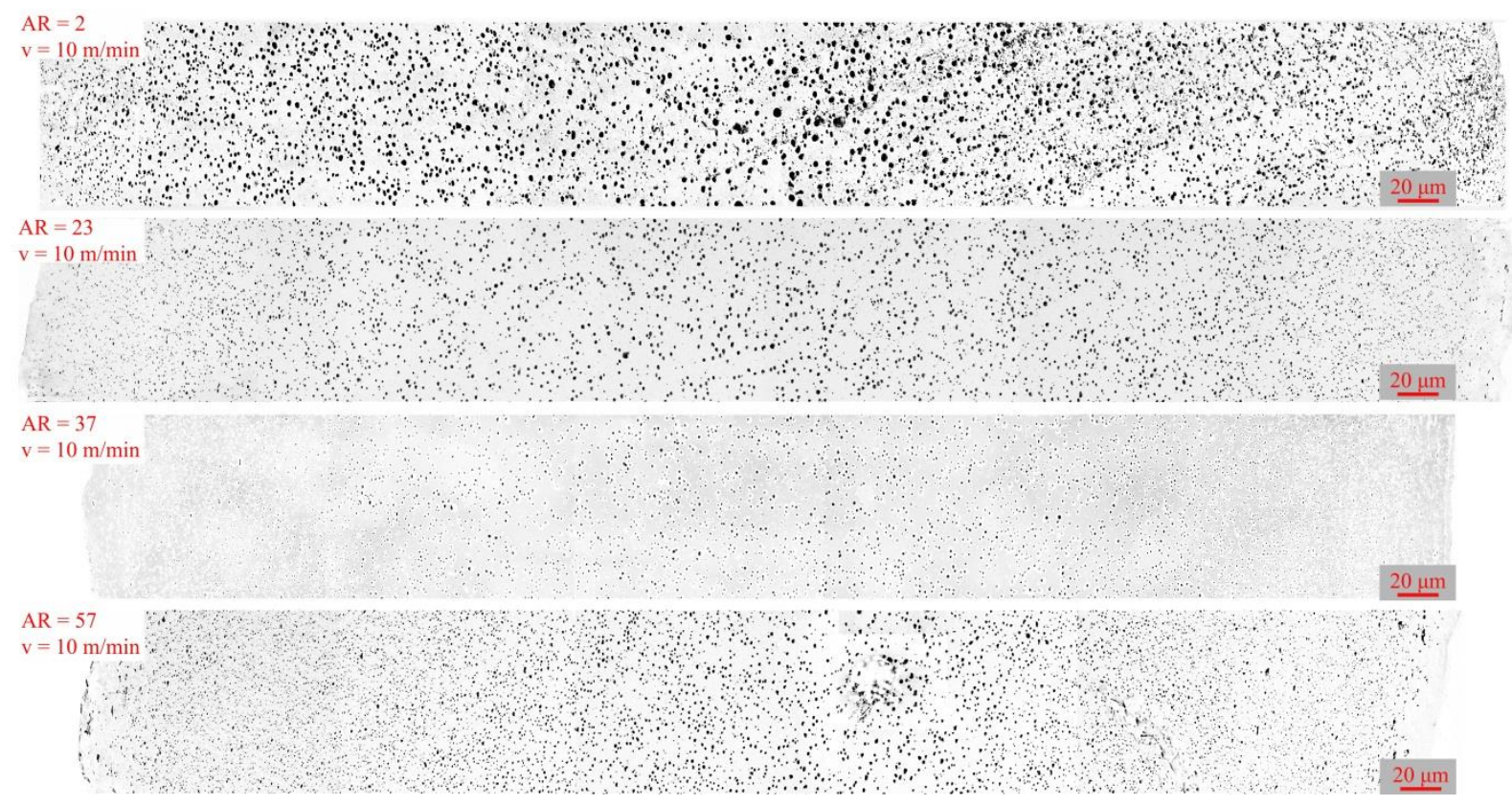

Figure 3. Combined SEM micrographs representing cross-sections of fibers produced with different capillary ARs and take-up speeds v.

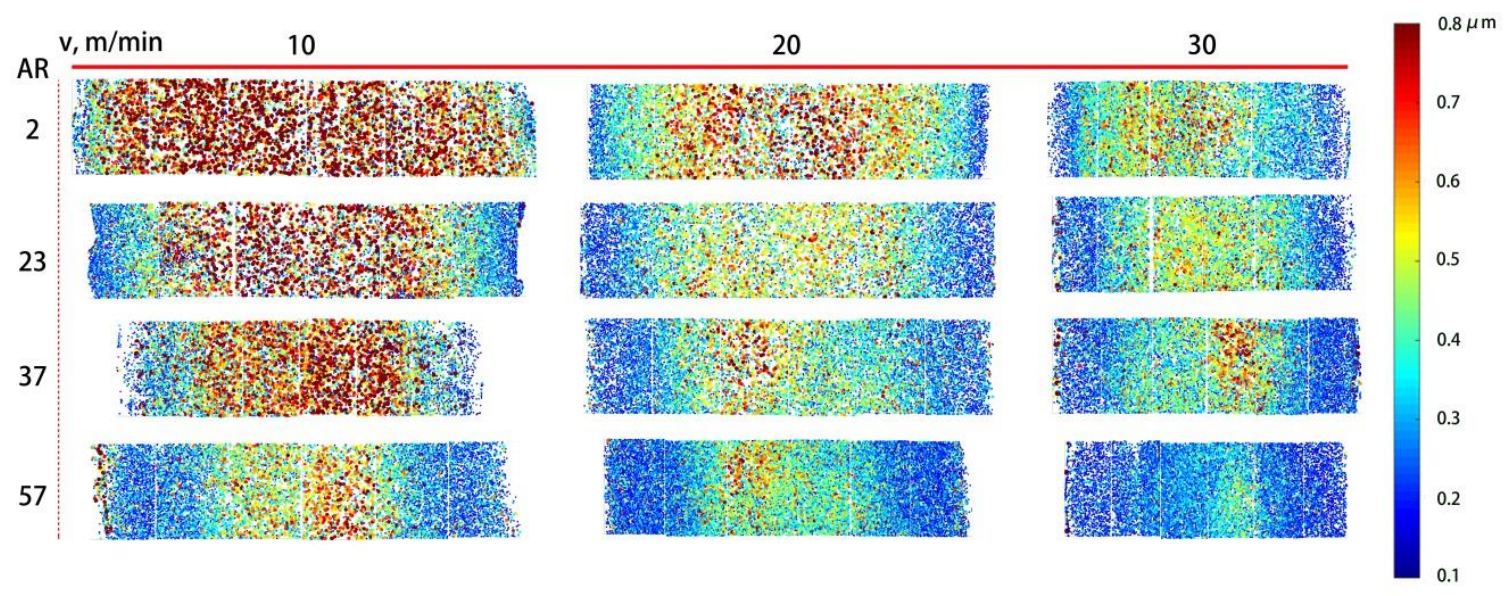

Figure 4. Morphology analysis of cross-sections of PP/PS fibers produced with different capillary ARs and take up speeds v. Each colored point is representing a PS droplet, where the color corresponds to a certain droplet radius according to the given color bar.

The gradient structures were further investigated regarding occurrence frequencies (in terms of the number probability parameter defined in Equation (2)) of large and small droplets dispersed across the fiber cross-section, see Figure 5. The capillary AR and the take-up speed $\mathrm{v}$ clearly control the morphology formation as demonstrated by the developed gradient 
patterns. In the case of capillary with $A R=2$ and take-up speed $v=10 \mathrm{~m} / \mathrm{min}$, almost no gradient morphology is observed in the cross-section. In contrast, gradient structures emerge dramatically with an increase of the capillary AR, at an unchanged take-up speed of $10 \mathrm{~m} / \mathrm{min}$. This observation can be explained by the longer exposure period to the parabolic shearing velocity profile that a blend experiences in longer capillaries. The take-up speed, on the other hand, slightly shifts the size gradient in the fiber axis to smaller droplet sizes. This observation is explained by the direct relationship between take-up speed and elongational forces which can oblong droplets and fibrils independent of their radial position. ${ }^{[27]}$ These competing features in some cases can lead to a large population of small dispersed domains, accompanied by a small population of large islands at different locations. Furthermore, the results indicate that the probability of relatively large droplets (radius $r>0.4 \mu \mathrm{m})$ to occur decreases with increasing take-up speed, i.e. with increasing elongational forces. 


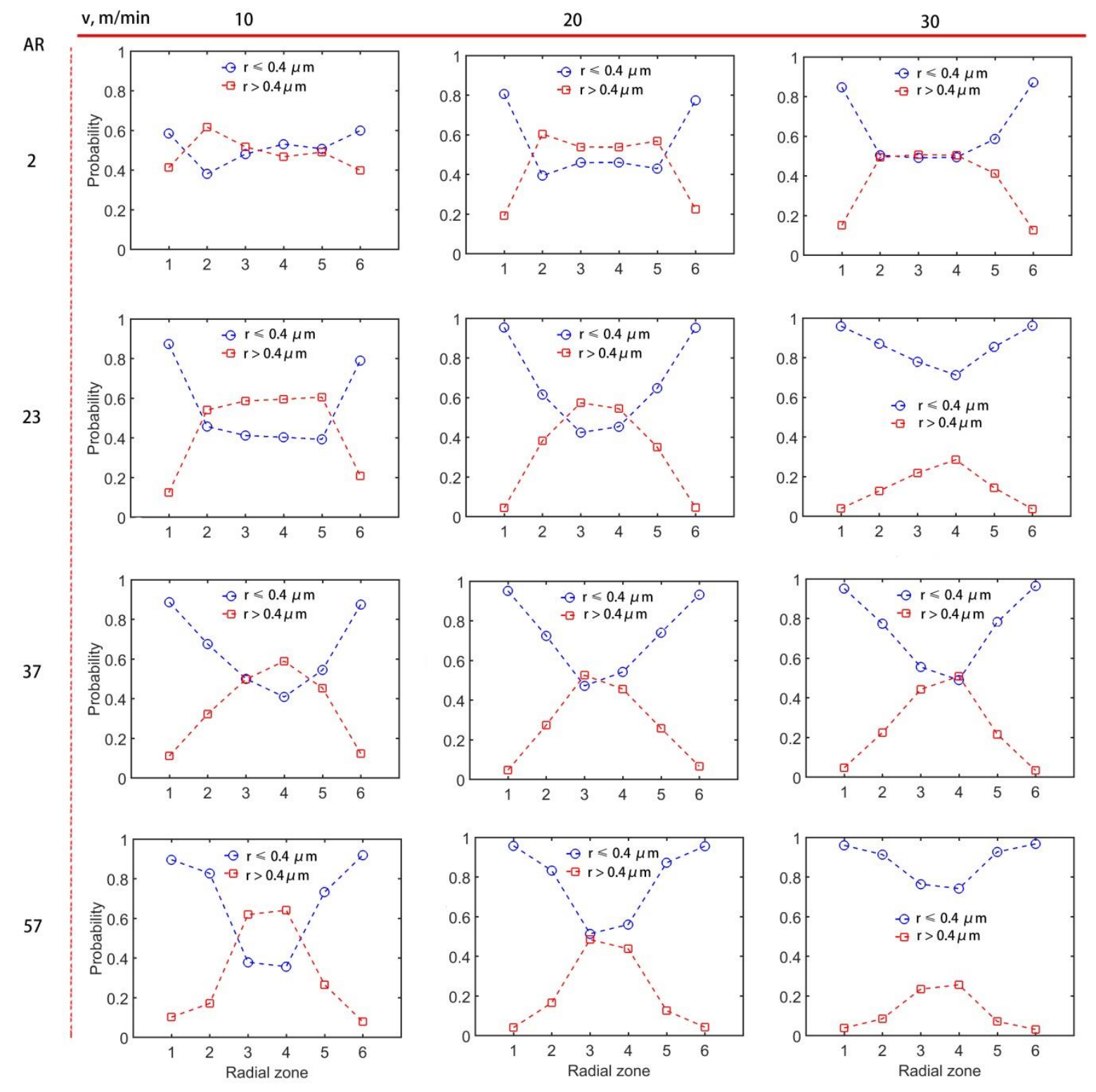

Figure 5. Number probability distribution of PS domains in the fiber cross-section, with different radius ranges of $r \leq 0.4 \mu \mathrm{m}$ (blue) and $r>0.4 \mu \mathrm{m}$ (red). Note that $0.4 \mu \mathrm{m}$ is a good approximation of the mean droplet radius measured in the central zone (Figure S1).

In addition to the cross-sectional gradient microstructures (in terms of droplet radii), longitudinal fiber sections are investigated to provide a full picture of the formed gradient morphology. Figure 6 shows the combined micrographs of longitudinal sections of PP/PS blend fibers (cut in half), melt-spun at different conditions. PS dispersed domains appear as fibrils with small diameters near the surface region, and occur as deformed droplets with large diameters near the central region. The PS fibrils become thinner and more stretched 
with increasing take-up speed and capillary AR. Considering the gradient morphologies observed in the fiber cross-sections (Figure 3), the decrease of droplet radii in cross-sectional cuts can mainly be attributed to the stretching of droplets. More interestingly, the observations suggest that the developed gradient morphology is not merely a gradient of sizes, but also a gradient of microstructures, i.e. from deformed droplets to stretched fibrils.

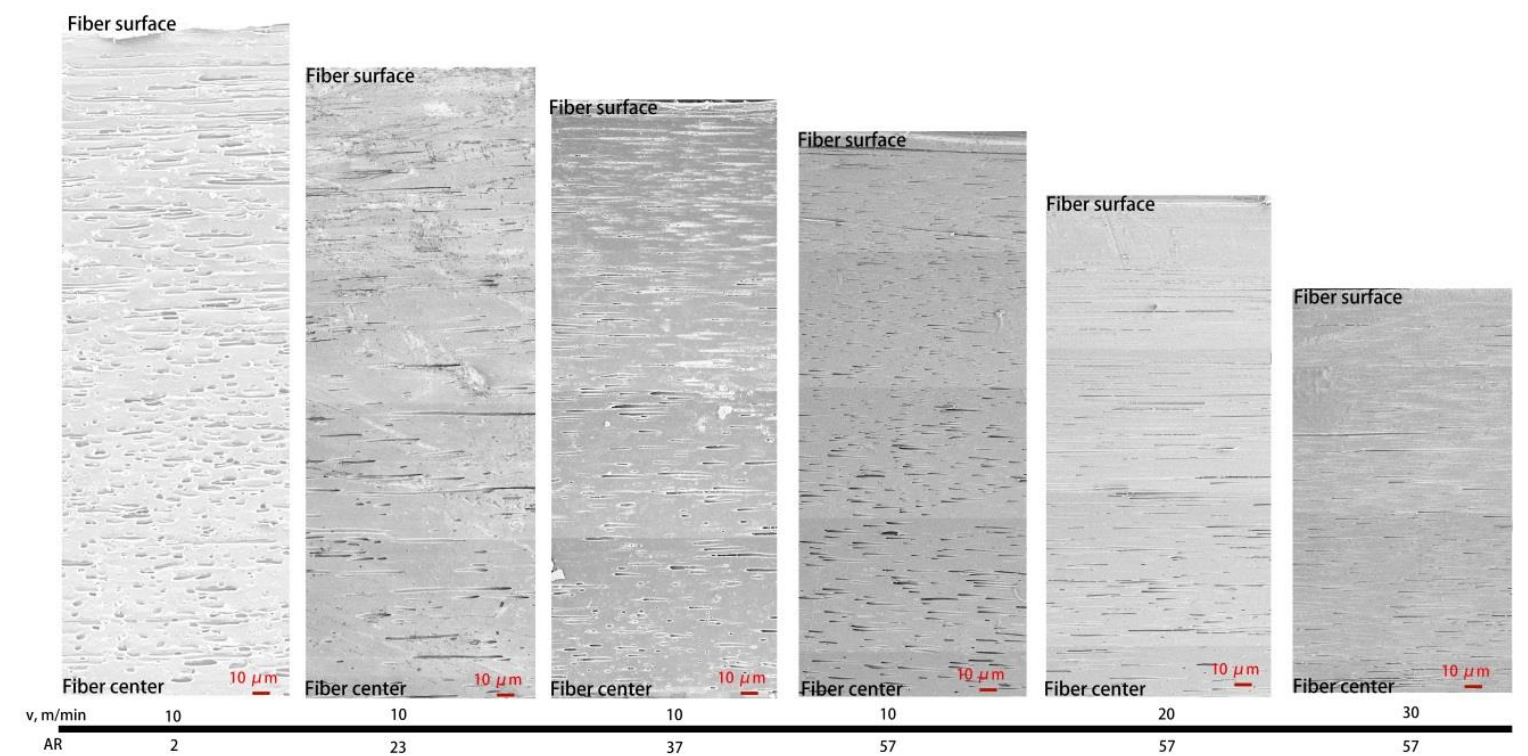

Figure 6. SEM micrographs of (half) longitudinal sections of PP/PS blend fibers produced with different capillary ARs and take-up speeds v.

When PP/PS blends are melt-pressed through a capillary die, the polymer chains experience both elongational and shear stresses in the entry zone due to converging flow with the elongational component dominating the flow. ${ }^{[22]}$ Dispersed droplets will be elongated into ellipsoidal, rod-like or fibrillar shapes in this region. Subsequently, the deformed dispersed phases will pass through the capillary die, where deformation, retraction, breakup and coalescence of dispersed domains can happen when respective conditions are satisfied. After the blend exists the capillary, it is mainly controlled by the elongational force of the take-up action. Therefore, the final morphology of PP/PS blends results from a combination of shear 
and elongational flows in different stages of the extrusion and drawing process. Hence, the process parameters can be designed in order to achieve a desired gradient morphology for a specific purpose. The processing conditions are summarized in Table 1 (and in more detail in Table S1). The residence time for polymer melts flowing through the shortest capillary die is only $\sim 0.05 \mathrm{~s}$, which is typically too short for a further deformation of dispersed droplets. ${ }^{[7]}$ Therefore, the morphology of the blends in the die with the smallest AR can be representative of the morphology developed in entrance and (to a small extent) take-up zone only.

The deformation of droplets in the take-up process is proportional to the draw-down ratio (DDR). DDR is $\sim 3$ at the take-up speed of $10 \mathrm{~m} / \mathrm{min}$. However, the shape factor ( $\mathrm{SF}=$ average aspect ratio, defined for the dispersed PS domains) of dispersed domains estimated from Figure 6 at the central part of the fiber ( $A R=2$ and $v=10 \mathrm{~m} / \mathrm{min}$ ) is $\sim 13 \pm 7$. Despite the large number of microscopy images used to measure SFs of the PS domains, the SFs reported here tend to fall below actual SFs due to a tilt angle of the fibrils with respect to the cut. In any case, SFs of the dispersed domains are significantly larger than DDR indicating that the impact of draw-dawn on stretching and elongation of the PS domains is relatively small, compared to the combination of large shear and elongational deformations in die entry zone. The same analysis applies to other processing conditions and dies showing that the gradient morphologies are mainly formed before the take-up zone. In order to further investigate the influence of the take-up zone, the distance between spinneret and water surface d was varied between 5 and $20 \mathrm{~cm}$ (respective processing parameters are summarized in Table S1 of the Supporting Information). Longer distances d provide more time for the 
molten fibrils and deformed droplets to stretch before quenching in water. However, these investigations did not reveal any specific trend in the morphology developments by varying d (Figures S2 to S6 of the Supporting Information). Consequently, the length of the take-up zone (exemplified here in terms of d) does not systematically influence the gradient morphology development within the limits and conditions studied here. Still, the take-up speed alters shape and size gradients of the morphology as discussed before. As a result, the main elongational deformation in the take-up zone already occurs within $5 \mathrm{~cm}$ after the die exit where the melt strand thins and adjusts itself to the take-up speed. This region is the effective elongational region of the take-up zone due to velocity changes in the drawing direction. Therefore, the take-up zone refines and stretches the dispersed domains, despite not being responsible for creating the gradient morphology in the first place. However, in extreme cases of high take-up speeds, it is expected that the gradient morphology disappears due to the uniaxial deformation of all domains regardless of their radial position. ${ }^{[27]}$

The combined effect of entry zone and capillary die gets even stronger when AR is increased inducing gradient microstructures in the blend. For dies with larger ARs, residence times are considerably longer. This might impose complex microstructural evolutions as typically found in emulsions, including droplet coalescence and breakup. ${ }^{[49-51]}$ While the former can increase the size of the PS domains, the latter phenomenon can utterly diminish the gradient morphology by breaking up elongated fibrils into smaller dispersed domains. The SEM micrographs depicted in Figure 6 show that the stretched long PS fibrils close to the surface appear to break up more frequently with increasing AR at a maintained take-up speed. In the morphology evolution of polymer blends, the importance of droplet breakup is considered 
higher when the droplets have a micron and/or submicron size, ${ }^{[22,27]}$ whereas coalescence becomes important for smaller sizes. ${ }^{[51]}$ In the following section, we investigate the formation mechanism of the gradient morphology in the dies.

\subsection{Formation Mechanism for Gradient Morphology}

The morphology of polymer blends critically depends on the rheological properties of the polymers, particularly on their viscosity ratio. The viscosity ratio plays a vital role in the micro-rheological behavior of liquid-liquid emulsions and eventually determines the matrixdispersed morphology of polymer blends during processing. ${ }^{[16,18,19]}$ Figure 7 shows the viscosities and the viscosity ratio of the polymers versus shear rate at $230{ }^{\circ} \mathrm{C}$. Both $\mathrm{PP}$ and PS show typical shear thinning behavior. The viscosity of PS is higher than that of PP, and the viscosity ratio of PS to PP is between 2 to 3 in the tested shear rate range. The zero shear viscosity $\left(\eta_{0}\right)$ of the polymers was obtained through regression using the three-parameter Bird-Carreau model. ${ }^{[52]}$

$$
\eta=\frac{\eta_{0}}{\left(1+(\lambda \dot{\gamma})^{2}\right)^{\frac{1-n}{2}}}
$$

where $\eta$ is the shear viscosity, $\eta_{0}$ the zero shear viscosity, $\dot{\gamma}$ the shear rate, $\mathrm{n}$ the power law index, and $\lambda$ the relaxation time. The zero shear viscosities of PP and PS are calculated to be $\sim 655$ and $\sim 1617 \mathrm{~Pa} \cdot \mathrm{s}$, respectively, and the zero shear viscosity ratio is $\sim 2.5$. The other fitted parameters of Equation 4 are given in Table S2 of the Supporting Information. A high viscosity ratio (typically greater than 1 ) is believed to be beneficial to obtain a fibrous dispersed phase in polymer blends. ${ }^{[16,53]} \mathrm{A}$ viscosity ratio larger than 1 can add an important property to the blend, i.e. the possibility to design gradient morphologies with varying size 
and shape of the dispersed phase. Further rheological characterization of the polymers using strain amplitude and frequency sweep experiments are provided in the Supporting Information, Figures S7 and S8.
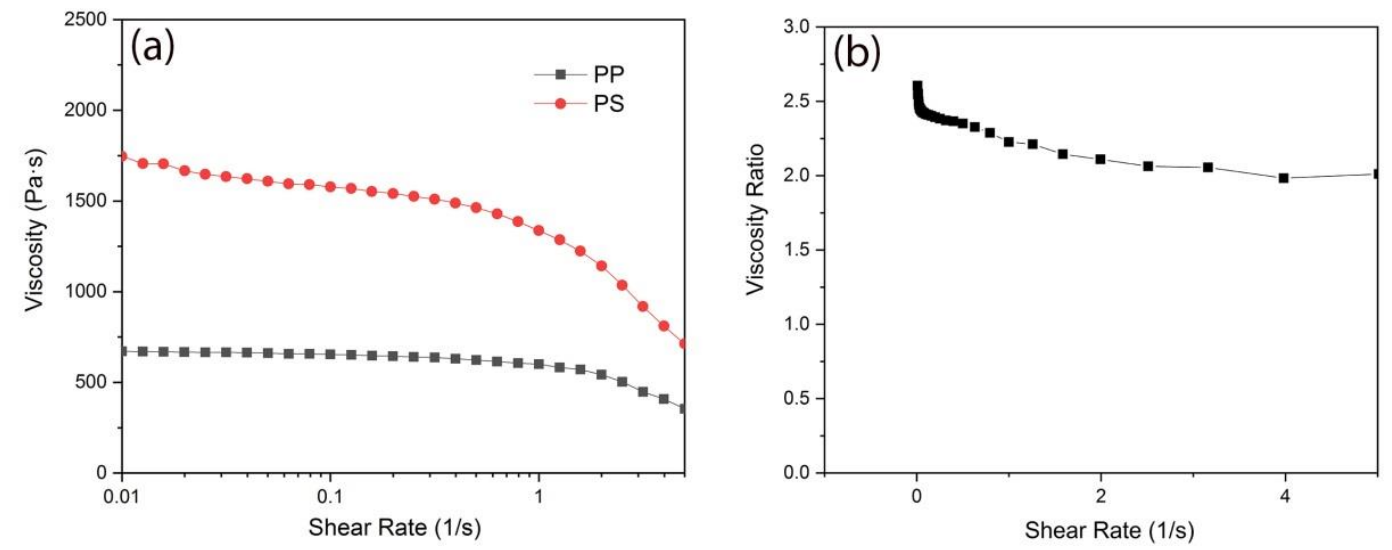

Figure 7. The shear viscosities of PP and PS (a), and the viscosity ratio of PS to PP (b) as a function of shear rate.

Formation mechanisms of gradient structures can be deducted easier when the effect of breakup can be ignored. ${ }^{[54]}$ In principle, the deformation and breakup of dispersed droplets can be estimated by Taylor's theory. ${ }^{[4,55]}$ In his theory, the capillary number $\mathrm{Ca}$ is defined as the ratio of viscous forces (trying to deform the droplet) and interfacial tension (trying to preserve the shape of the droplet):

$$
\mathrm{Ca}=\frac{\eta_{\mathrm{m}} \dot{\gamma} \mathrm{R}_{0}}{\alpha}
$$

where $\eta_{m}$ is the matrix viscosity, $\dot{\gamma}$ the shear rate, $R_{0}$ the original droplet radius, and $\alpha$ the interfacial tension of polymer blends. $\mathrm{R}_{0}$ is estimated $\sim 4 \mu \mathrm{m}$ from the average volume of the central zone in Figure 6. The polymer viscosity was calculated from Equation (4). Droplet deformation and breakup depend on the reduced capillary number $\mathrm{Ca}^{*}{ }^{* 7]}$ : 


$$
\mathrm{Ca}^{*}=\frac{\mathrm{Ca}}{\mathrm{Ca}_{\mathrm{c}}}
$$

The critical capillary number $\mathrm{Ca}_{\mathrm{c}}$ in shear flow is defined as:

$$
\log C a_{c}=-0.506-0.0994 \log p+0.124 \log ^{2}(p)-\frac{0.155}{\log p-0.611}
$$

where $\mathrm{p}$ is the respective viscosity ratio. The breakup time $t_{b}$ for the dispersed phase can be calculated from Utracki's empirical equation ${ }^{[50]}$ :

$$
\mathrm{t}_{\mathrm{b}}=84 \mathrm{p}^{0.345}\left(\mathrm{Ca}^{*}\right)^{-0.559}
$$

$\mathrm{Ca}^{*}$ and $\mathrm{t}_{\mathrm{b}}$ in the different dies were calculated and employed to explain the behavior of the dispersed domains in shear flows as shown in Table 2. The value of $\mathrm{Ca}^{*}$ at the die wall indicates that the shearing stresses are stronger than the critical conditions necessary to deform and (possibly) break up polymer droplets $\left(\mathrm{Ca}>\mathrm{Ca}_{\mathrm{c}}\right)$. On the other hand, $\mathrm{t}_{\mathrm{b}}$ is much larger than the mean residence time in the capillary dies. Therefore, the majority of the dispersed PS domains should further deform into fibrils without breaking up by the action of shearing forces.

Table 2. Capillary number and breakup time for dispersed domains at the die wall.

\begin{tabular}{lllllll}
\hline & & & Critical \\
Capillary & $\begin{array}{l}\text { anterfacia } \\
1 \text { tension } \\
{[\mathrm{mN} / \mathrm{m}]}\end{array}$ & $\begin{array}{l}\text { Capillary } \\
\text { number } \\
{[\mathrm{Ca}]}\end{array}$ & $\begin{array}{l}\text { Reduced } \\
\text { capillary } \\
\text { number } \\
{\left[\mathrm{Ca}_{\mathrm{c}}\right]}\end{array}$ & $\begin{array}{l}\text { capillary } \\
\text { number } \\
{\left[\mathrm{Ca}^{*}\right]}\end{array}$ & $\begin{array}{l}\text { Time for } \\
\text { breakup, } \\
\mathrm{t}_{\mathrm{b}}[\mathrm{s}]\end{array}$ & $\begin{array}{l}\text { Mean } \\
\text { residence } \\
\text { time in } \\
\text { capillary } \\
\text { die }[\mathrm{s}]\end{array}$ \\
\hline 2 & & 10.4 & & 6.94 & 31.0 & 0.05 \\
23 & 4.64 & 10.1 & \multirow{2}{*}{1.50} & 6.74 & 31.4 & 0.67 \\
37 & 9.9 & & 6.60 & 31.7 & 1.10 \\
57 & 9.8 & & 6.53 & 31.9 & 1.70 \\
\hline
\end{tabular}

anterfacial tension is calculated from Palmer's equation. ${ }^{[56]}$ 
A more precise consideration of the radial distribution of shear stresses in capillary flow is necessary considering the radius-dependent gradient morphology. It is well known that the pressure-driven velocity profile is parabolic in a capillary die, with the highest shear rate near the wall and zero shear rate at the centerline (Figure 8a). In order to understand the radial differences in morphology, the radial profiles of the $\mathrm{Ca}^{*}$ and $\mathrm{t}_{\mathrm{b}}$ are plotted in Figure $8 \mathrm{a}$ and $\mathrm{b}$, respectively, for different capillary dies. The increasing value of $\mathrm{Ca}^{*}$ from the centerline to the wall indicates that it is more probable for droplets to deform into fibrils and break up near the wall. The comparison between $t_{b}$ and the residence time profile of blends in the radial direction $t_{r}$ also suggests that the droplets closer to the wall have more time to undergo deformation and breakup. Therefore, only a small portion of the droplets located very close to the surface of the fibers will deform into long fibrils and eventually break up. This behavior becomes more significant as the AR increases. For a range of initial droplet sizes $R_{0}$ of $0.1-100 \mu \mathrm{m}$, the region where $t_{r}>t_{b}$ is limited to a fiber surface layer with the thickness of $\sim 2-60 \mu \mathrm{m}$. The estimated thickness range of this layer is in agreement with the morphologies in Figure 6. This shows that the probability of both fibril formation and breakup is considerably higher in the surface region of the fibers. SEM images of the cross section close to the walls illustrate that the number density of PS domains increases with increasing capillary AR (Figure 9). This observation confirms the prediction that both dominance of fibril formation and occurrence of breakup in the surface region increase with the AR of the capillary dies. Based on the capillary theory, dispersed domains apart from the surface region do not break up, and mainly deform into longer fibrils as the blends flow through capillary dies. Indeed, the dispersed domains in the central region of the die 
experience less deformation than those at the surface. Consequently, a gradient morphology is built up within the bulk of the material as a result of the shear flow velocity gradient in radial direction.
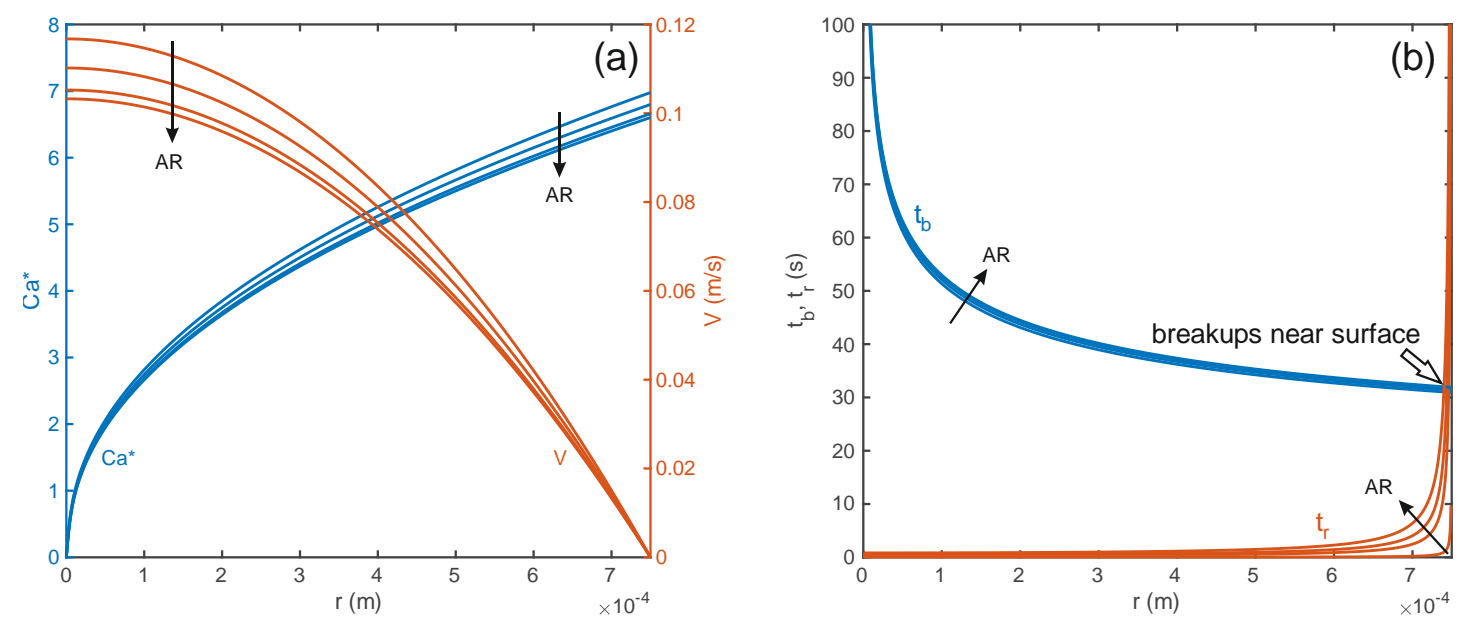

Figure 8. (a) Reduced capillary number $\mathrm{Ca}^{*}$, and velocity profile along the capillary axis $\mathrm{V}$ as a function of the radial position $r$. The velocity profile is calculated using the parabolic equation $V(r)=\frac{2 Q}{\pi R^{2}}\left(1-\left(\frac{r}{R}\right)^{2}\right)$ based on momentum balance, where $Q$ and $R$ are the volumetric flowrate and the capillary radius, respectively. ${ }^{[57]}$ (b) Radial distributions of required time for breakup $t_{b}$, and residence time $t_{r}$. Curves are plotted for different die capillary ARs, with arrows indicating increasing ARs. The theory predicts that the droplets close to the surface can undergo breakup, while the droplets at the central region do not have enough time for it. Note that $t_{r}=\frac{L}{V(r)}$ with $L$ being the length of capillary dies.

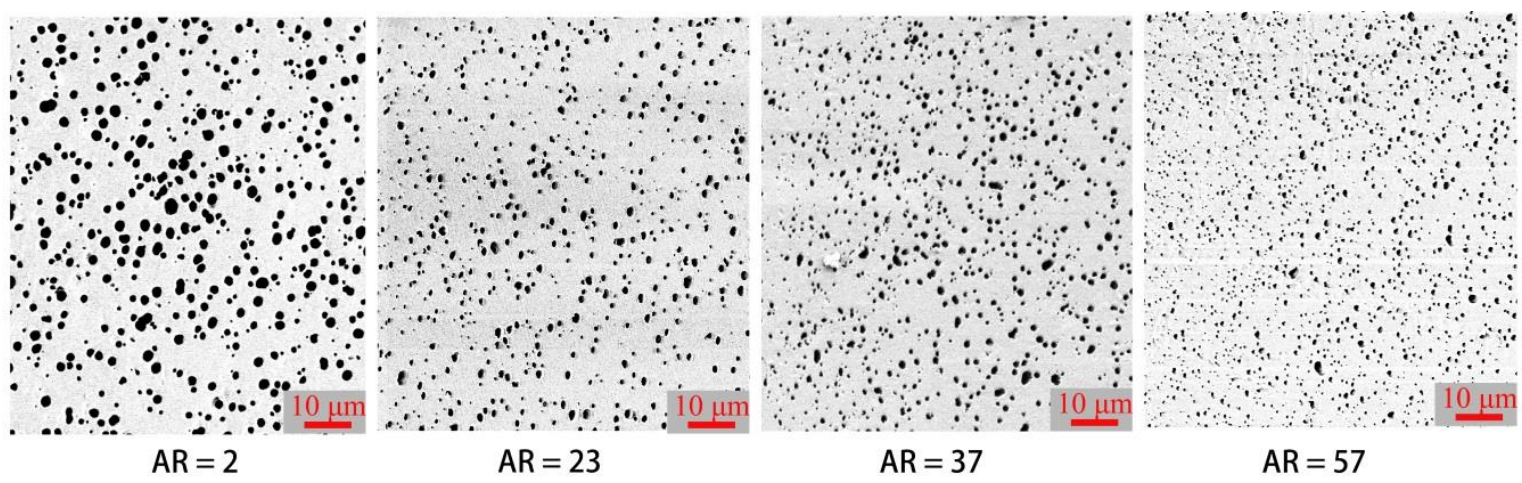

Figure 9. Cross-section SEM micrographs (taken from the second radial partition) of PP/PS blend fibers, melt-spun with different capillary ARs and a take-up speed of $10 \mathrm{~m} / \mathrm{min}$. 


\subsection{Anisotropic Surfaces of PP/PS Blends}

According to the results presented so far, the gradient morphologies of the blends show completely different microstructures at the surface of the fibers than in their bulk. The formation of stretched dispersed fibrils at the surface enables modified surface properties without changing the bulk properties (of the matrix polymer). The possibility to dynamically manipulate surface features by flow rheology is indeed desirable. The realized surface morphologies of the fibers are thus further studied in this section. For this purpose, the PS phase is dissolved and washed away from the surface to reveal the microstructure. Furthermore, the effect of hot-drawing (as a post-processing step) on the refinement of surface patterns is investigated.

The surface micrographs of PP/PS blend fibers after dissolving PS are shown in Figure 10. Sub-micrometer channels aligned along the fiber axis are observed on the surface. These channels are influenced by the variation of take-up speed and/or capillary AR. The gradient microstructure with fine ordered fibrils in the surface region and large deformed droplets in the bulk can be controlled effectively by the design of the capillary die. According to the results, elongated fibrils are formed more easily in the capillary with the largest AR and/or at the highest take-up speed. In this respect, reversed gradient structures can also be fabricated by tuning rheology (e.g. viscosity ratio), die design (e.g. AR), and processing parameters (e.g. take-up speed). Yang et.al ${ }^{[30]}$ described the morphology of PP/ethylenebutene copolymer $(\mathrm{EBM})$ blend $(\mathrm{p}=0.35$ with $20 \%$ weight percent of dispersed EBM) extruded through a capillary die $\left(\mathrm{D}_{\text {cap }}=2 \mathrm{~mm}, \mathrm{AR}=0.23\right.$ and $26^{\circ}$ entrance angle $)$, and found 
a gradient structure with longer EBM fibrils in the core and elongated droplets in the surface region. Therefore, one can imagine a variety of novel functional polymeric materials with desired morphological structures and surface (sub)micro-patterns which could be fabricated in a continuous process.

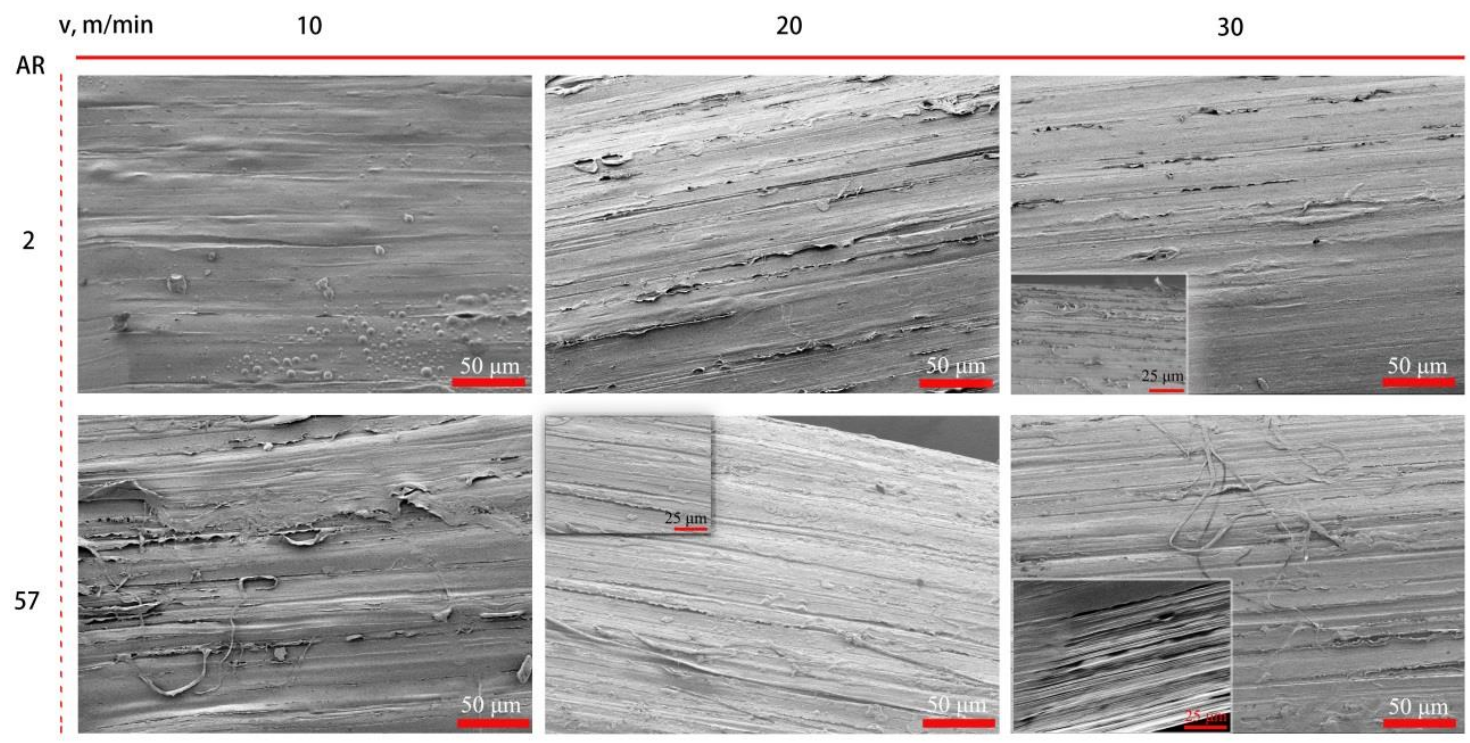

Figure 10. SEM micrographs of the gradient fiber surfaces after dissolving PS. The fibers are melt-spun with different capillary ARs and a take-up speeds $\mathrm{v}$.

The Fourier transforms of representative SEM micrographs indicate the anisotropic patterns at the surfaces where the submicron channels (of dissolved PS fibrils) are oriented in the axial direction of the fiber, see Figure 11. The surface PS fibrils produced by the die with smallest AR are scarce; however, their corresponding textures are anisotropic and aligned in the direction of the fiber axis. These patterns become more frequent and refined as the AR of the die is increased. The anisotropy is also intensified as evidenced by the Fourier transform of the surface microstructure. Exploiting the effect of the capillary AR, i.e. the role of the shear flows, to develop submicron (even nanosized) surface topologies is very promising. The gained knowledge is easily transferable to design, for instance, thin films, 
offering a vast field of high-tech applications such as optical polymer thin films ${ }^{[44]}$ and polymer microelectronics. ${ }^{[45]}$ The relevance is further supported by the similarity of size scales and anisotropy of the realized textures regarding surface patterns needed for such applications. In contrast, conventional methods, mainly based on lithographic techniques and/or spinodal dewetting, offer surface patterns with a higher uniformity. ${ }^{[43]}$ This, however, can be improved in the continuous production method shown here by adopting an optimized mixture of shear/elongational flows and/or post-processing steps such as hot-drawing.
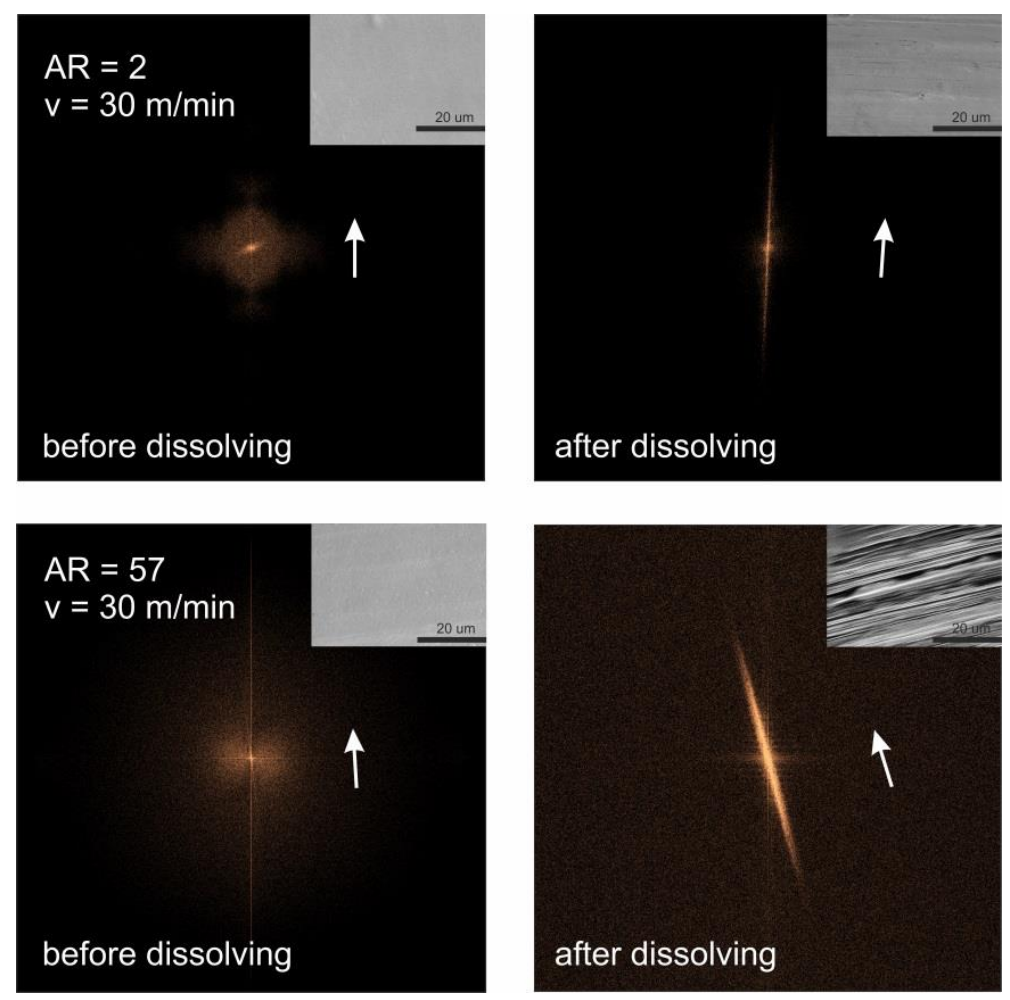

Figure 11. Fourier transforms of the representative SEM micrographs (insets) of the fiber surfaces before (left column) and after (right column) dissolving PS from the surface. The upper and lower rows show the surfaces of the fibers processed with $\mathrm{v}=30 \mathrm{~m} / \mathrm{min}$ and $\mathrm{AR}=2$ and 57, respectively. In each picture, the direction of the fiber axis is indicated with a white arrow.

A hot-drawing process is applied to further refine the anisotropic surface and to achieve continuous submicron (or nanosized) surface patterns. The surfaces of hot-drawn fibers are 
investigated utilizing AFM technique, see Figure 12. The fiber surface patterns become more prominent with increasing capillary AR. Submicron channels with $\sim 0.2-1 \mu \mathrm{m}$ in width and $\sim 0.2-1.5 \mu \mathrm{m}$ in height are aligned along the fiber axis. The surface morphologies of gradient fibers after hot-drawing are shown in Figure S9 of the Supporting Information, along with their corresponding Fourier transforms. A comparison of the surface patterns before and after hot-drawing suggests that the textures are refined by the post-processing, with final characteristic dimensions in the order of $\sim 100 \mathrm{~nm}$. Furthermore, the anisotropy is intensified by hot-drawing as indicated by the more pronounced alignment patterns in the Fourier transforms. It should be noted that some PP fibrils might peel off from the fiber surface due to the high drawing force, and/or exfoliate from the surface due to the removal of PS fibrils. SEM micrographs of such PP fibrils formed by hot-drawing with higher draw-ratios are shown in Figure S10 of the Supporting Information. This finding presents a limitation in the design of such materials using the current process/post-process approach, if finer textures are required. One possibility to overcome this problem is to increase the take-up speed during processing in order to refine the surface fibril formation online. This would at the same time increase the processing speed. Future studies are necessary in this direction to ensure that such an approach is practical. 

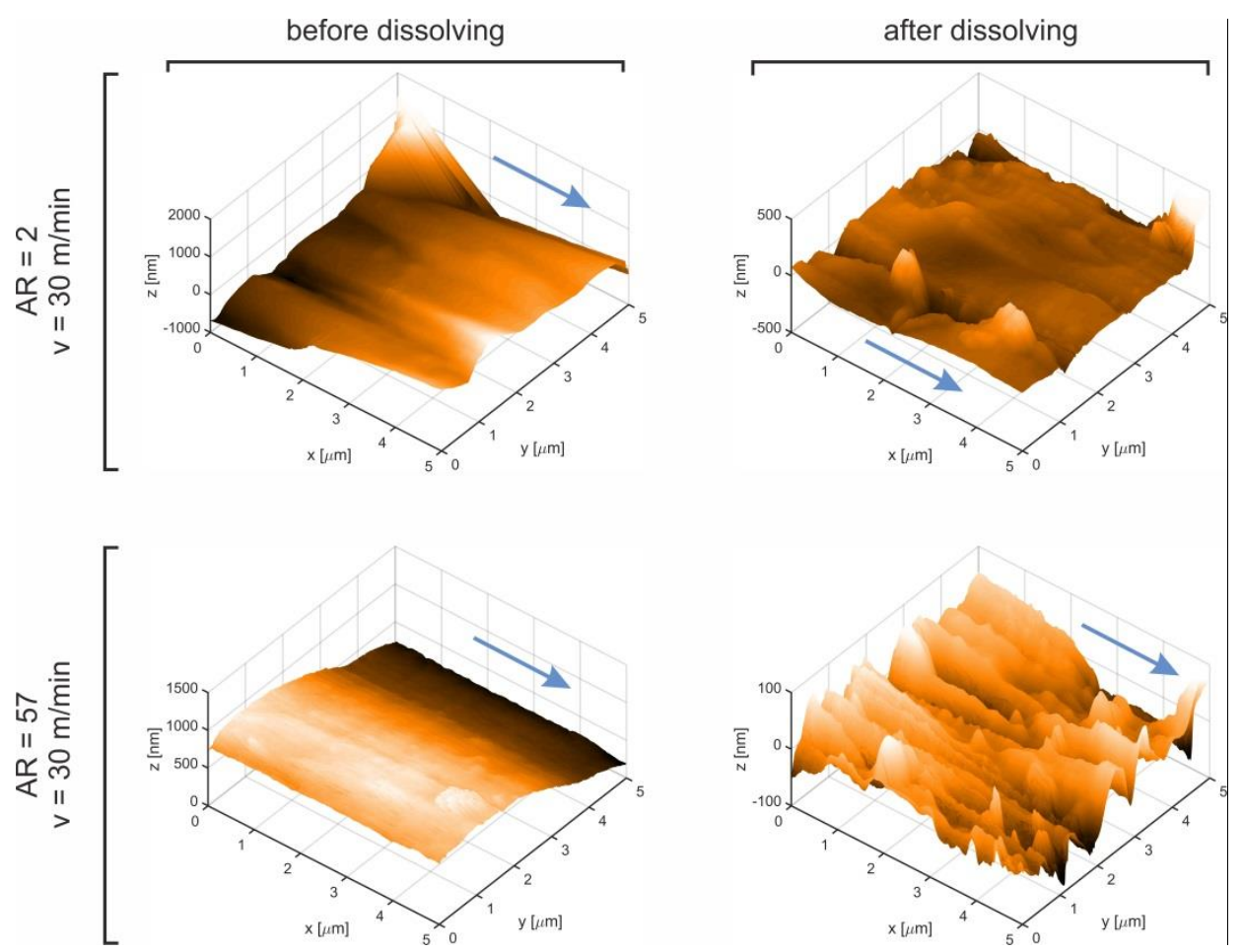

Figure 12. AFM images of the surface patterns of gradient fibers after hot-drawing, before (left column) and after (right column) dissolving PS. The upper and lower rows show the surfaces of the fibers processed with $\mathrm{v}=30 \mathrm{~m} / \mathrm{min}$ and $\mathrm{AR}=2$ and 57, respectively.

Arrows indicate the fiber axis.

\section{Conclusions}

Gradient polymer blends were prepared (by melt-spinning) with fine fibrils in the surface region and large deformed droplets in the central region. The gradient features could be manipulated by the variation of capillary AR and take-up speed. The average cross-sectional droplet radius decreased with increasing take-up speed and, to a lesser extent, with increasing capillary AR. However, with larger ARs, the polymer blends near the capillary wall experienced a longer exposure to shear, due to the parabolic velocity profile in the capillary die. This allowed droplets near the fiber surface to deform into fibrils or to break up. The take-up speed, on the other hand, slightly shifts the occurrence probability from larger to smaller PS domain cross-sections due to stretching. In sum, it was shown that the gradient 
microstructure in the bulk can be effectively controlled by die design (e.g. capillary AR), and processing parameters (e.g. take-up speed). Submicron (nanosized) channels aligned along the fiber axis are observed on the surface of gradient fibers after dissolving the dispersed phase. The elongated fibrils are facilitated by capillaries with high AR and/or high take-up speeds. It was shown that this anisotropic morphology can be further improved by a hot-draw post-processing, in order to achieve finer and more ordered elongated fibrils on the surface of blend fibers. The results can lead to a novel approach to continuously produce submicron patterned surfaces.

\section{Acknowledgements}

Dan Pan acknowledges financial support by the China Scholarship Council. We also thank Mathias Lienhard and Thomas Niessen for their assistance in the experiments.

\section{References}

[1] B. Hexig, H. Alata, N. Asakawa, Y. Inoue, Adv. Funct. Mater. 2005, 15, 1630.

[2] X.-M. Xie, Y. Chen, Z.-M. Zhang, A. Tanioka, M. Matsuoka, K. Takemura, Macromolecules 1999, 32, 4424.

[3] S. Amada, MRS Bull. 1995, 20, 35.

[4] J. Park, K. Char, C.-W. Park, Ind. Eng. Chem. Res. 1999, 38, 4675.

[5] Y. Koike, Polymer 1991, 32, 1737.

[6] Y. Koike, T. Ishigure, E. Nihei, J. Light. Technol. 1995, 13, 1475.

[7] Q. Xing, M. Zhu, Y. Wang, Y. Chen, Y. Zhang, J. Pionteck, H. J. Adler, Polymer 2005, 46, 5406 .

[8] Q. Shen, P. Shi, M. Gao, X. Yu, Y. Liu, L. Luo, Y. Zhu, Mater. Sci. Eng. C 2013, 33, 1860 .

[9] K. F. Leong, C. K. Chua, N. Sudarmadji, W. Y. Yeonga, J. Mech. Behav. Biomed. 
Mater. 2008, $1,140$.

[10] Q. Tran-Cong, J. Okinaka, Polym. Eng. Sci. 1999, 39, 365.

[11] L. Zhu, Y. Wang, X. Yu, X. Shen, X. Xu, J. Porous Mater. 2015, 22, 119.

[12] B. Hexig, H. Alata, Y. Inoue, J. Polym. Sci. Part B Polym. Phys. 2005, 43, 3069.

[13] L. M. Bronstein, A. Ivanovskaya, T. Mates, N. Holten-Andersen, G. D. Stucky, J. Phys. Chem. B 2009, 113, 647.

[14] H. R. Thomas, J. J. O’Malley, Macromolecules 1979, 12, 323.

[15] H. R. Thomas, J. J. O’Malley, Macromolecules 1981, 14, 1316.

[16] Y.-J. Gao, Z.-Y. Liu, C.-L. Yin, S.-L. Huang, M.-B. Yang, Polym. Adv. Technol. 2012, 23, 695.

[17] A. Gooneie, C. Holzer, Polymer 2017, 125, 90.

[18] A. Gooneie, H. Nazockdast, F. Shahsavan, Polym. Eng. Sci. 2015, 55, 1504.

[19] A. Gooneie, H. Nazockdast, F. Shahsavan, Polym. Eng. Sci. 2016, 56, 51.

[20] U. Sundararaj, C. W. Macosko, R. J. Rolando, H. T. Chan, Polym. Eng. Sci. 1992, 32, 1814.

[21] D. Bourry, B. D. Favis, Polymer 1998, 39, 1851.

[22] F. Oosterlinck, I. Vinckier, M. Mours, H. M. Laun, P. Moldenaers, Rheol. Acta 2005, $44,631$.

[23] C. Lacroix, M. Grmela, P. J. Carreau, J. Nonnewton. Fluid Mech. 1999, 86, 37.

[24] U. Sundararaj, Y. Dori, C. W. Macosko, Polymer 1995, 36, 1957.

[25] A. Soroudi, M. Skrifvars, J. Appl. Polym. Sci. 2011, 119, 2800.

[26] K. B. Migler, Phys. Rev. Lett. 2001, 86, 1023.

[27] M. Heindl, M.-K. Sommer, H. Münstedt, Rheol. Acta 2004, 44, 55.

[28] Y. Yan, A. Gooneie, H. Ye, L. Deng, Z. Qiu, F. A. Reifler, R. Hufenus, Macromol. Mater. Eng. 2018, 303, 1800214.

[29] A. A. Leal, O. A. Neururer, A. Bian, A. Gooneie, P. Rupper, K. Masania, C. Dransfeld, R. Hufenus, Polymer 2018, 142, 375.

[30] J. Yang, J. L. White, Q. Jiang, Polym. Eng. Sci. 2010, 50, 1969.

[31] A. Rizvi, Z. K. M. Andalib, C. B. Park, Polymer 2017, 110, 139.

[32] N. H. A. Tran, H. Brünig, R. Boldt, G. Heinrich, Polymer 2014, 55, 6354. 
[33] S. Fakirov, Compos. Sci. Technol. 2013, 89, 211.

[34] X.-M. Xie, M. Matsuoka, K. Takemura, Polymer 1992, 33, 1996.

[35] X.-M. Xie, T.-J. Xiao, Z.-M. Zhang, A. Tanioka, J. Colloid Interface Sci. 1998, 206, 189.

[36] K.-W. D. Lee, P. K. Chan, X. Feng, Chem. Eng. Sci. 2004, 59, 1491.

[37] T. Nguyen-Dang, A. C. de Luca, W. Yan, Y. Qu, A. G. Page, M. Volpi, T. Das Gupta, S. P. Lacour, F. Sorin, Adv. Funct. Mater. 2017, 27, 1605935.

[38] R. Bagherzadeh, M. Gorji, M. Latifi, P. Payvandy, L. X. Kong, Fibers Polym. 2012, $13,529$.

[39] M. L. Brongersma, Y. Cui, S. Fan, Nat. Mater. 2014, 13, 451.

[40] M. Sun, M. McGowan, P. J. Kingham, G. Terenghi, S. Downes, J. Mater. Sci. Mater. Med. 2010, 21, 2765.

[41] I. I. Stoyanova, R. J. A. van Wezel, W. L. C. Rutten, J. Neural Eng. 2013, 10, 066018.

[42] I. Tonazzini, E. Jacchetti, S. Meucci, F. Beltram, M. Cecchini, Adv. Healthc. Mater. 2015, 4, 1849.

[43] E. Huovinen, M. Suvanto, T. A. Pakkanen, J. Micromechanics Microengineering 2015, 25, 055005.

[44] M. Ibn-Elhaj, M. Schadt, Nature 2001, 410, 796.

[45] A. M. Higgins, R. A. L. Jones, Nature 2000, 404, 476.

[46] N. Widjojo, T.-S. Chung, D. Y. Arifin, M. Weber, V. Warzelhan, Chem. Eng. J. 2010, $163,143$.

[47] K. Gupta, P. Chokshi, R. Stepanyan, J. Nonnewton. Fluid Mech. 2016, 230, 1.

[48] MATLAB, http://www.mathworks.com/2017.

[49] S. Guido, Curr. Opin. Colloid Interface Sci. 2011, 16, 61.

[50] L. A. Utracki, Z. H. Shi, Polym. Eng. Sci. 1992, 32, 1824.

[51] M. A. Huneault, Z. H. Shi, L. A. Utracki, Polym. Eng. Sci. 1995, 35, 115.

[52] T. Osswald, N. Rudolph, Polymer Rheology: Fundamentals and Applications, Carl Hanser Verlag, Munich 2015.

[53] N. Chapleau, B. D. Favis, J. Mater. Sci. 1995, 30, 142.

[54] H. P. Grace, Chem. Eng. Commun. 1982, 14, 225. 
[55] G. I. Taylor, Proc. R. Soc. A Math. Phys. Eng. Sci. 1932, 138, 41.

[56] G. Palmer, N. R. Demarquette, Polymer 2005, 46, 8169.

[57] R. B. Bird, R. C. Armstrong, O. Hassager, Dynamics of Polymeric Liquids, John Wiley \& Sons Inc., New York 1987. 